\title{
(6) OPEN ACCESS \\ Acute tear of the fascia cruris at the attachment to the Achilles tendon: a new diagnosis
}

\author{
Nick Webborn, ${ }^{1}$ Dylan Morrissey, ${ }^{2}$ Kasthuri Sarvananthan, ${ }^{2}$ Otto Chan ${ }^{3}$
}

${ }^{1}$ Centre for Sport and Exercise Science and Medicine (SESAME), University of Brighton, Eastbourne, UK ${ }^{2}$ Centre for Sports and Exercise Medicine, Bart's and the London School of Medicine and Dentistry, Queen Mary University of London, Mile End Hospital, London, UK ${ }^{3}$ BMI London Independent Hospital, London, UK

\section{Correspondence to}

Dr Nick Webborn, The Centre for Sport and Exercise Science and Medicine (SESAME), University of Brighton, Eastbourne, UK. nickwebborn@sportswise.org. uk

Accepted 19 August 2014 Published Online First 8 September 2014

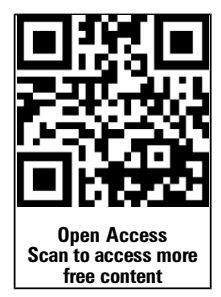

\section{CrossMark}

To cite: Webborn $\mathrm{N}$ Morrissey D,

Sarvananthan $\mathrm{K}$, et al. $\mathrm{Br} J$ Sports Med 2015;49:

1398-1403.

\section{ABSTRACT}

Background The fascia cruris encloses the posterior structures of the calf and connects to the paratenon and the Achilles tendon. We describe the clinical

presentation, ultrasound imaging characteristics and the time to the recovery of tears of the fascia cruris at the attachment to the Achilles tendon.

Methods Retrospective review of 11 tears of the fascia cruris in the different legs as separate events in 9 patients ( 6 male and 3 female, mean age 35.52 years, range 11-48) identified using diagnostic ultrasound, after presenting with Achillodynia.

Results 11 participants presented at a mean of 4.5 weeks (range $0.5-12$ ) after onset of symptoms. The left Achilles was more commonly injured than the right $(7: 4)$ and the lateral side more than the medial $(6: 4)$ with one case with medial and lateral presentation. Clinically, there was swelling and tenderness over the medial or lateral border in the mid to upper portion of the Achilles. 7 of the 11 (63.6\%) had functional overpronation. Ultrasound appearances of a tear were identified as hypoechoic area extending from the medial or lateral border of the Achilles extending along the anatomical plane of the fascia cruris. Average return to activity was 5.2 weeks (range 1-22). Participants presenting later had longer recovery but all participants returned to full activity $(r=0.4)$.

Conclusions This is the first description of the clinical details and sonographic findings of a tear to the fascia cruris at its attachment to the Achilles tendon. This needs to be considered as a cause of Achillodynia in athletes as recognition will affect the management.

\section{INTRODUCTION}

The fascia cruris is that layer of connective tissue that encloses all the posterior structures of the calf down to the ankle joint and connects to the Achilles tendon. The Achilles tendon lacks a true synovial sheath but instead has a paratenon on the dorsal, medial and lateral sides between the fascia cruris and the Achilles tendon (see figure 1). Pain in the Achilles tendon region is a common problem in the competitive athlete and in the general population. ${ }^{12}$

Injury to the fascia cruris at the attachment to the Achilles has not before been considered as a cause of Achillodynia. The fascia cruris facilitates small yet significant sliding movements of its collagen layers, ensuring the fascia can deform to accommodate the underlying muscle morphology but also withstand the high tensile forces generated during locomotion. ${ }^{3}$ The fascia cruris has an intimate relationship with the paratenon and the Achilles tendon, and as a load bearing structure, it has the potential to be injured. Figure 2A,B shows an anatomical dissection and a microscopic section of the attachment of the crural fascia to the paratenon. It is evident from these images not only how thin these structures are, and the intimate relationship between the paratenon and the fascia cruris, but also the potential for tearing under mechanical load. Tears of the fascia cruris have recently been observed on ultrasound in clinical practice and are clinically relevant to diagnosing Achilles pain accurately and are discussed in this article.

A review of the literature highlighted the lack of evidence detailing the role of the fascia cruris and only one report suggested its pathological significance $^{3-6}$; this is complemented by anatomical descriptions or its surgical release in the treatment of Achilles peritendinitis. ${ }^{7-9}$ The crural fascia has a mean thickness of $924 \mu$ and consists of three layers of parallel collagen fibre bundles separated by a thin layer of loose connective tissue. The collagen fibre arrangement gives the crural fascia anisotropic characteristics, i.e. the properties are directionally dependent, strongly suggesting alignment along lines of force.

In an anatomical dissection study of the fascia cruris and the Achilles tendon, ${ }^{10}$ the fascia cruris and the paratenon overlying the Achilles were continuous structures. ${ }^{10}$ These authors suggested that it might be implicated in a 'progenitor stage' of Achilles tendinopathy mediated by inflammation at the interface of the fascia and the tendon. However, conversely, a combined anatomical and radiological study showed that in some participants, these layers could be dissected and distinguished as separate entities. The confluence between the fascia cruris and the paratenon was discernible at a mean anatomical distance of $37.3 \mathrm{~mm}$ (range 17-58) proximal to the posterosuperior calcaneal tubercle ${ }^{4}$. Figure 3 illustrates the confluence in longitudinal and transverse plane and biomechanically represents the point most likely to be injured.

High-resolution MRI studies have shown the paratenon to be a distinct layer more proximally but fusion with the fascia cruris then occurring distally and the layers becoming indistinct. ${ }^{5}$ Historically, the operative treatment of chronic paratenonitis has been described with division of the crural fascia on both sides of the tendon from the paratenon. ${ }^{11}$ This suggests that the tension between the two structures is implicated in the development of paratenonitis or at least necessary to be divided for the paratenon to heal.

We describe a case series of athletes with pain in the Achilles region that we have identified as having tears of the fascia cruris from the attachment to the paratenon and the Achilles tendon 


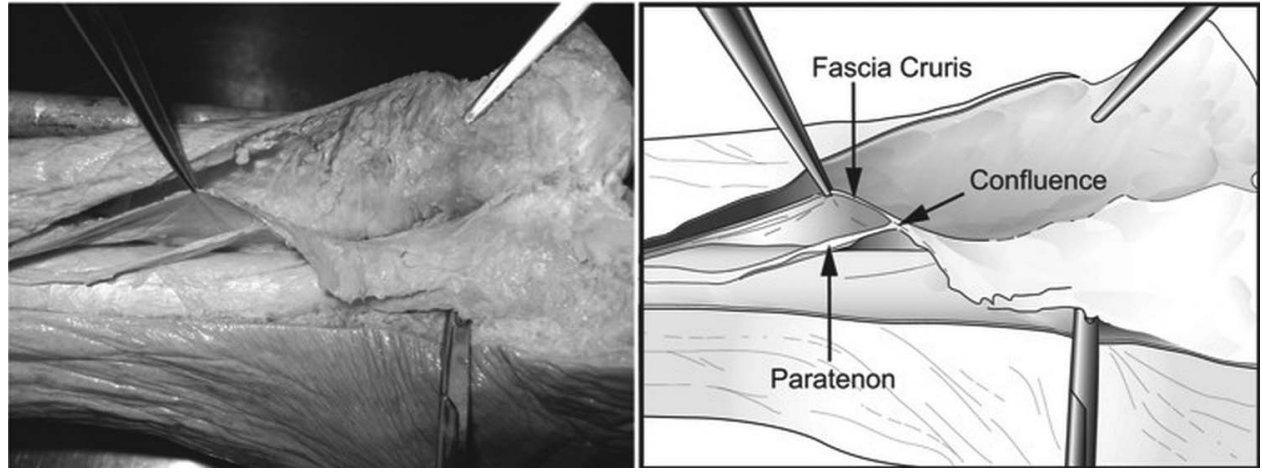

Figure 1 Anatomical dissection revealing the confluence of the fascia cruris and the paratenon and the relationship to the posterosuperior calcaneal tubercle. From Carmont et al 2011 with permission.

using diagnostic ultrasound and discuss the possible causes, treatment and outcomes.

\section{METHODS}

A retrospective review of a series of patients presenting with pain in the Achilles tendon region to a single sports injury clinic between the periods December 2008 and December 2012 was performed to select patients who were identified to have tears of the fascia cruris at the attachment to the Achilles tendon. The patient record review was approved by the University of Brighton, Ethics and Governance Committee. The study
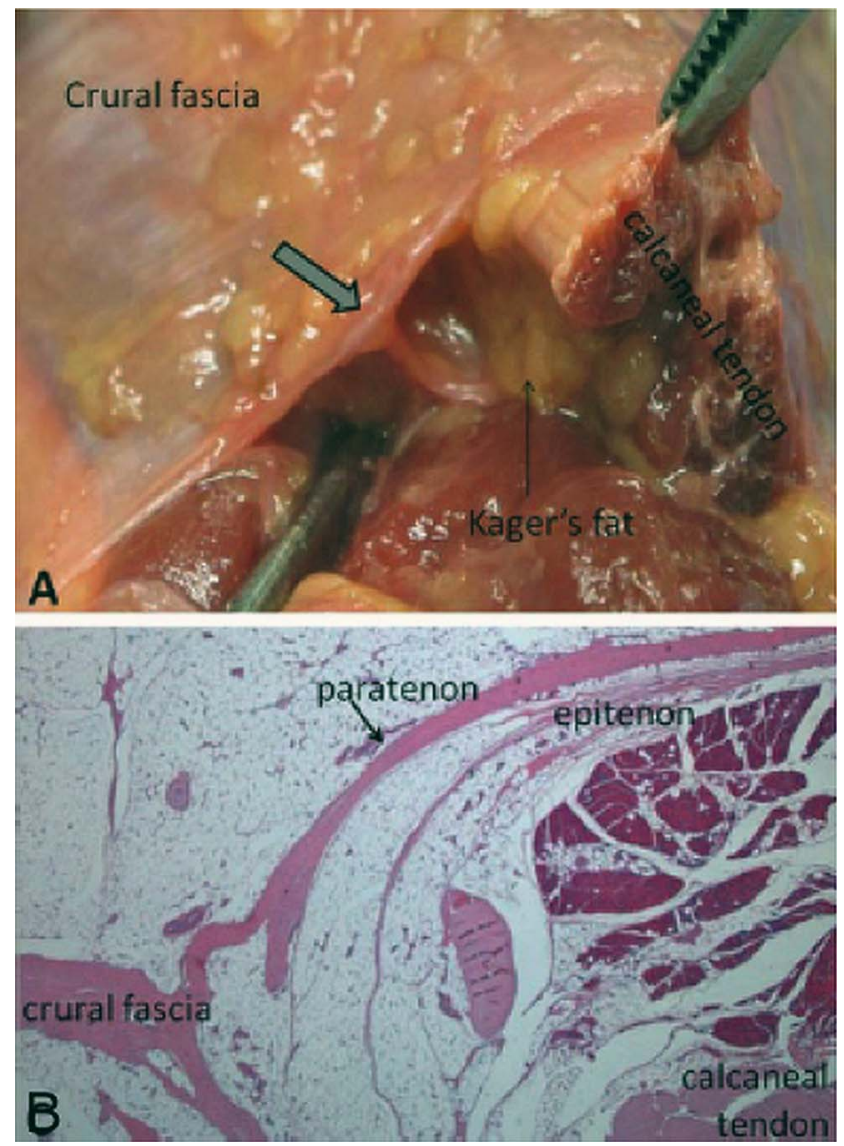

Figure 2 (A) Anatomical dissection and (B) microscopic section of the attachment of the crural fascia to the paratenon. Images supplied by Antonio Stecco from University of Padua, Padua, Italy. included 9 patients $(6$ male and 3 female, mean age 35.52 years, range 11-48) among whom 11 tears of the fascia cruris were documented. Two patients had developed tears in the contralateral limb as a separate event several months apart.

The patient notes and ultrasound images were retrospectively assessed to identify details of symptom history, clinical findings, foot biomechanics and sporting participation. The treatment and time for return to sporting activity was also noted. As a retrospective audit of clinical cases identified with a particular pathology, no patient consent or ethical approval was required and after extracting data for analysis the participants were de-identified.

The diagnosis was made on diagnostic ultrasound (Sonosite MicroMaxx-Sonosite, Inc, Bothell, Washington, USA) with a linear probe $(13-6 \mathrm{MHz})$ using the examination protocol for the Achilles as per the European Society of Musculoskeletal Radiology. ${ }^{12}$ The imaging was performed by an experienced sports physician with over 12 years experience in musculoskeletal sonography, with a special interest in Achilles pathology. The tear characteristics were identified as areas of hypoechoic change at the medial or lateral attachment to the Achilles tendon in the short axis view which extended anteriorly in the anatomical plane of the fascia cruris and that the paratenon on the dorsal surface of the tendon was normal. The relationship with the musculotendinous junction (MTJ) was noted along with any evidence of neovascularisation within the area of the tear. The presence of any accompanying typical findings of Achilles tendinopathy or other pathology was also noted. The images were also independently reviewed remotely by the fourth author, a highly experienced musculoskeletal radiologist.

\section{RESULTS}

The nine patients were typically highly active participants comprising four sub-3 h marathon runners, three high-level triathletes and two tennis players (see table 1). The patients were seen and assessed at a mean of 4.95 weeks (range 0.5-12) after the onset of symptoms. The left Achilles was more commonly involved than the right $(7: 4)$ and the lateral side of the Achilles was involved in six cases compared with four cases on the medial side. One patient had changes both medially and laterally.

Typically patients reported a sensation of calf tightness over the preceding days or weeks and then a rapid onset of pain in the Achilles region during activity. The onset would usually accompany a rapid increase in the training load or change in the training type. In two patients, there was a history of a sudden ankle inversion moment as the initiating factor. One patient 
A
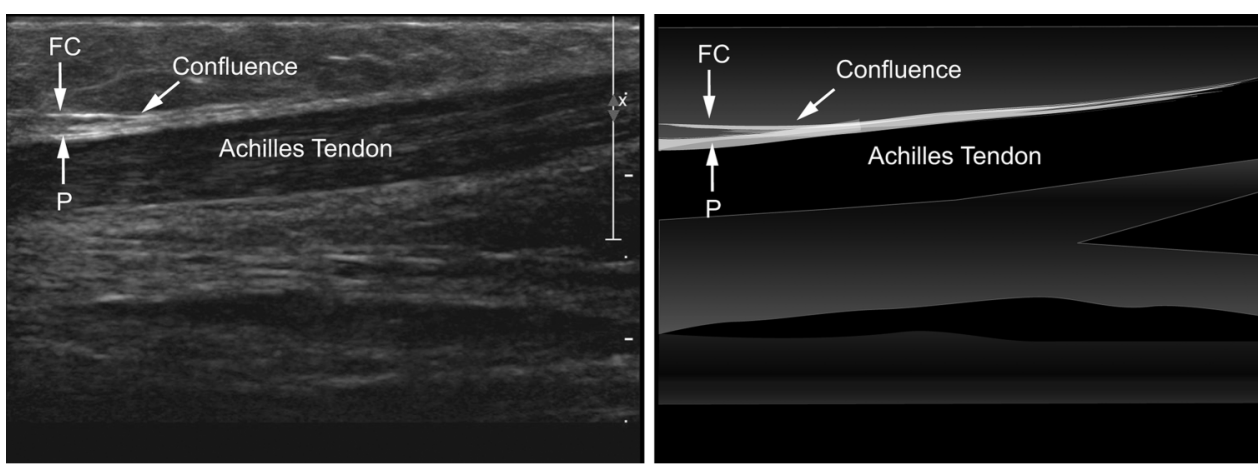

$\mathrm{B}$
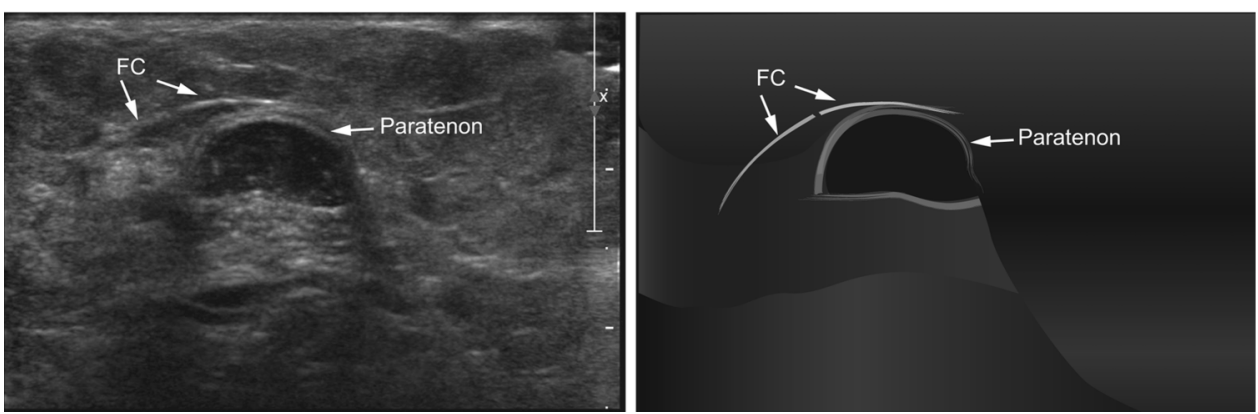

Figure 3 (A) Longitudinal ultrasound image illustrating the confluence of the layers of the fascia cruris ( $\mathrm{FC}$ ) and the paratenon (P). (B) Transverse ultrasound image illustrating separate layers of the layers of FC and P. From Carmont et al 2011 with permission.

described the onset of symptoms as a sensation 'like a bee sting' at the Achilles. Other factors included performing a new jumping exercise in a gym routine, running on uneven terrain in old shoes, dancing vigourously in high heels and wearing new trainers in a 10000 race. They all reported a sudden onset of pain and tenderness over the medial or lateral border of the Achilles associated with swelling, and two cases developed sensation of crepitus. The patient aged 11 incurred two episodes of

Table 1 Participant information of fascia cruris tears by age, gender, sport, activity at onset, side, biomechanics and location

\begin{tabular}{|c|c|c|c|c|c|c|}
\hline Age & Gender & Sport & Mechanism & Side & Biomechanics & $\begin{array}{l}\text { Med/ } \\
\text { Lat/ } \\
\text { both }\end{array}$ \\
\hline $20^{*}$ & $\mathrm{M}$ & Triathlon & $\begin{array}{l}\text { Ankle } \\
\text { inversion }\end{array}$ & $\mathrm{L}$ & Pronator & Lat \\
\hline $20 *$ & M & Triathlon & Nil specific & $\mathrm{R}$ & Pronator & Lat \\
\hline 28 & $\mathrm{~F}$ & Triathlon & $\begin{array}{l}\text { Felt pain on } \\
\text { take off }\end{array}$ & $\mathrm{R}$ & Pronator & Med \\
\hline $46^{*}$ & $\mathrm{~F}$ & Marathon & $\begin{array}{l}\text { Dancing in } \\
\text { high heels }\end{array}$ & $\mathrm{R}$ & Pronator & Med \\
\hline $46^{*}$ & $\mathrm{~F}$ & Marathon & $\begin{array}{l}\text { Variation in } \\
\text { terrain }\end{array}$ & $\mathrm{L}$ & Pronator & Med \\
\hline 41 & $\mathrm{~F}$ & Marathon & Nil specific & $\mathrm{L}$ & Pronator & Lat \\
\hline 45 & $M$ & Triathlon & Nil specific & $\mathrm{L}$ & Neutral & Med \\
\hline 47 & M & Marathon & Nil specific & $\mathrm{L}$ & Pronator & Lat \\
\hline 48 & $M$ & Tennis & Nil specific & $\mathrm{L}$ & Neutral & Lat \\
\hline 11 & $M$ & Tennis & $\begin{array}{l}\text { Ankle } \\
\text { inversion }\end{array}$ & $\mathrm{R}$ & Neutral & $\begin{array}{l}\text { Med } \\
\text { and Lat }\end{array}$ \\
\hline 28 & $M$ & Marathon & Nil specific & $\mathrm{L}$ & Neutral & Lat \\
\hline
\end{tabular}

inversion strain at the ankle while playing consecutive matches of tennis. The injury did not require him to stop play immediately but pain and swelling in the Achilles region developed soon after. Interestingly he had been treated just a few months previously for growth-related calf tightness. Two of the patients developed the same problem in the other leg within months of the other leg but as a distinct new problem.

Clinically there was mild swelling over the mid to upper portion of the Achilles tendon which was tender to the touch. Palpable crepitus was found in the two patients who reported the sensation. There was palpable tightness in the soleus and gastrocnemius in all cases. From a biomechanical perspective, 7 of the $11(63.6 \%)$ of the injured legs had functional overpronation.

\section{Ultrasound}

Diagnostic ultrasound identified findings compatible with a tear of the fascia cruris at the attachment to the Achilles tendon. The diagnosis was made when there was a hypoechoic area extending from the medial or lateral border of the Achilles extending anteriorly along the anatomical plane of the fascia cruris, that was associated with the area of clinical swelling and tenderness (see figure 4A). Since the fascia cruris measures less than $1 \mathrm{~mm}$ in thickness when a tear occurs, the over-riding feature is the hypoechoic area rather than distinct ends of the structures. Pressure with the probe reproduced the patients' symptoms. It was viewed in the short axis and required the probe to be tilted for adequate viewing of the fascia as per the European Society of Musculoskeletal Radiology technical guidelines for examination of the ankle. ${ }^{12} \mathrm{~A}$ side-by-side comparison view of injured to uninjured sides is helpful to the difference (see figure 4B). The hypoechoic area may be associated with neovascularisation when viewed with Power Doppler (see figure 4C). The dorsal surface of the paratenon generally appears normal, although 
there can be some change at the side of the attachment where the disruption occurs (see figure 4D). Two of the participants had sonographic evidence of coexisting changes of Achilles tendinosis with thickening of the tendon and decreased signal within the tendon body. In several of the participants, the extent of the tear extended superiorly for over $2 \mathrm{~cm}$, up to and including the MTJ.

\section{Treatment}

We report the treatment applied to the cases presenting; however, since these were novel presentations, we do not claim that this is necessarily the optimal regime. Treatment consisted of a combination of conservative measures including ice, relieving the tension in the triceps surae with acupuncture to soleus and gastrocnemius, offload taping, application of topical nonsteroidal gels or Traumeel S Gel (Heel GmbH, Baden-Baden, Germany), ${ }^{13}$ low-level therapeutic LASER $^{14}$ and soft tissue massage. A gradual stretching programme was followed later by strengthening and progressive loading once full pain-free stretch has been achieved, followed by sports-specific rehabilitation.

\section{Return to sport}

The average return to full activity was 5.2 weeks (range 1-22). One patient had two further recurrences of the injury after returning to full training with both episodes occurring about 3 months after the previous episode. Two patients went on to have ultrasound-guided peritendinous injections of lignocaine and hydrocortisone acetate $25 \mathrm{mg}$, as the symptoms had failed to settle adequately with conservative treatment. The injection was made under ultrasound guidance into the recess between the fascia and the Achilles. Patients not responding to the treatment, who required injection, tended to have a longer time to return to activity.

\section{DISCUSSION}

\section{Clinical presentation}

This is the first study presenting tears of the fascia cruris at the attachment to the Achilles tendon as an injury. The study shows that fascia cruris tears tend to be observed in highly active individuals who undergo unaccustomed or particularly strenuous activity. It may affect either gender and has been observed in a wide age range (11-48 years). Calf tightness appeared to be a risk factor for all patients, and it may be that calf tightness from intensive training or growth-related tension causes increased strain on the confluence between the fascia cruris and the paratenon which, when additionally subjected to increased loading, results in an acute tear. The history of symptoms is key in steering towards a diagnosis of fascia cruris tear to help differentiate from a paratenonitis; hence, the descriptions of a sudden onset of pain in an activity associated with localised swelling and tenderness on the medial or lateral border are typical of this injury. The tear can extend up to the MTJ in some cases involving the lower fibres of soleus and as such would be potentially classed as a type $3 \mathrm{~A}$ within the Munich consensus statement on the terminology and classification of muscle injuries in sport. ${ }^{15}$ This fits well with the clinical descriptors of a 'stabbing, sharp pain, often noticeable tearing at time of injury' and 'well-defined localised pain' and 'stretch-induced pain aggravation'.

This pattern of acute pain differs to that observed in Achilles tendinopathy which tends to develop more gradually or of paratenonitis which comes on acutely but not suddenly. In paratenontis there is a soreness over the posterior aspect of the Achilles and not only medially or laterally, and may be associated with friction of the tissue on the running shoe. The ultrasound changes are also typically on the dorsal superficial surface of the Achilles. There appear to be biomechanical risk factors whether it is simply overpronation or the speed of movement from pronation to supination or vice versa (see figure 5).

The tears on the lateral side could be due to the tension transmitted through the fascia as the foot pronates and supinates during ambulation. As the foot supinates to aid propulsion, the tension in the fascia cruris on the lateral side is exaggerated leading to tears during takeoff. A similar mechanism can be applied to tears on the medial side, which develop as tension mounts during pronation and sudden deceleration. Pre-existing calf muscle tension would exacerbate the tension on the confluence. The range for the confluence between the fascia cruris and the paratenon is reportedly quite wide $(17-58 \mathrm{~mm})$ and it is possible, and indeed likely from a biomechanical perspective, that the tear may start here and extend up or down. Figure $5 \mathrm{~A}$ shows the anatomical location of a tear on the medial side and the location of the fascia cruris. Figure $5 \mathrm{~B}$ shows how inversion and eversion of the foot can place a dynamic load on the confluence of the fascia cruris and the paratenon. This can be seen dynamically using sonography. The fascial 'sling' under extreme tension causes separation from the attachment to the medial or lateral portion of the Achilles.

\section{Ultrasound imaging features and tips}

The study has limitations in that it is a single centre retrospective study; however, the clinical and sonographic findings have been confirmed by some of the authors working from a different centre. The single clinician ultrasound operator was a very experienced sports physician with over 12 years experience in musculoskeletal sonography, and with a special interest in Achilles tendon pathology. ${ }^{16} 17$ The sonographic appearances did not fit in with any currently identified clinical pattern or previously described sonographic appearance.

For accurate scanning, the user must tilt the probe on each side of the tendon as the fascia cruris will run perpendicularly away from the probe in some positions and may be missed. Passive inversion and eversion of the foot is also recommended while imaging to show the different degrees of tension on the fascia. This may also bring on the patient's symptoms. Although no control can exist for a case series, we did image the uninjured contralateral limb for comparison. It is important to note the difference to findings in paratenonitis, which is usually noted as thickening of the paratenon on the dorsal surface of the Achilles that may extend to the medial and/or lateral border but would not extend anteriorly along the line of the fascia cruris.

Although two of the participants with fascia cruris tears also had sonographic signs of Achilles tendinopathy, it is unclear as yet whether these are related. Some theories have implicated the fascia cruris in the development of Achilles tendinopathy, a similar mechanism to that observed in plantar fasciitis. ${ }^{10}$ The authors suggested that if the fascia/tendon interface was the source of initial inflammation, then this could be the source of the pain seen in the early stage ('pre-reactive') in tendinopathy. Furthermore, they hypothesised that these changes could lead to the neovascularisation around the tendon.

\section{Management}

Treatments used so far have been typically those used in the management of soft tissue injuries and additionally addressing the underlying calf tightness and any biomechanical factors, but the ideal management protocol has yet to be determined. Consequently we describe the treatments applied and outcomes 

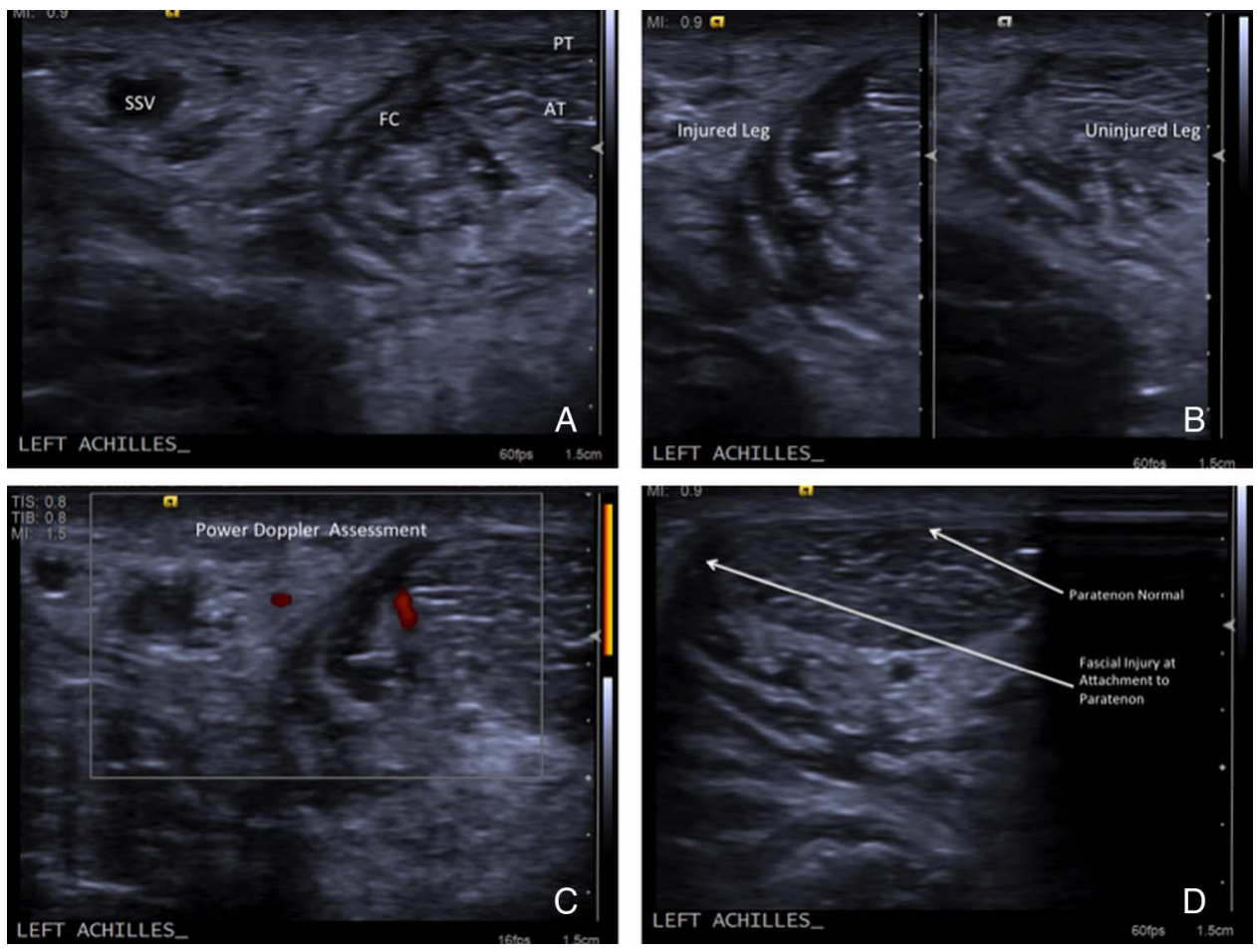

Figure 4 Transverse ultrasound images (A) showing the FC tear seen as hypoechoic area extending from the lateral border of AT. (B) Comparison images of injured and uninjured leg $(C)$ showing neovascularisation in the area of tear on Power Doppler and (D) showing normal PT on the dorsal surface and the area of injury at the attachment. AT, Achilles tendon; FC, fascia cruris; PT, paratenon; SSV, short saphenous vein.

in the cases seen (see table 2). We consider that if an acute tear is seen soon after injury then possibly immobilisation in a cast brace may be appropriate for a limited period but this is untested.

Participants who were taking longer to recover had an injection of a short-acting corticosteroid peritendinous injection under ultrasound guidance. These participants returned to activity on average 7 weeks after the injection. Our study design does not let us comment any further on whether corticosteroid injection is beneficial in this condition, but consideration of the risks and benefits of injection should be considered in the individual case; however, no reliable proof exists of the deleterious effects of peritendinous injections. ${ }^{18} 19$

\section{CONCLUSIONS}

The literature describing the fascia cruris is limited to its functional anatomy and its surgical release in the treatment of chronic paratenonitis. Injury to this structure has not been considered previously. Tears of the fascia cruris have been observed on ultrasound and are clinically relevant to diagnosing Achilles

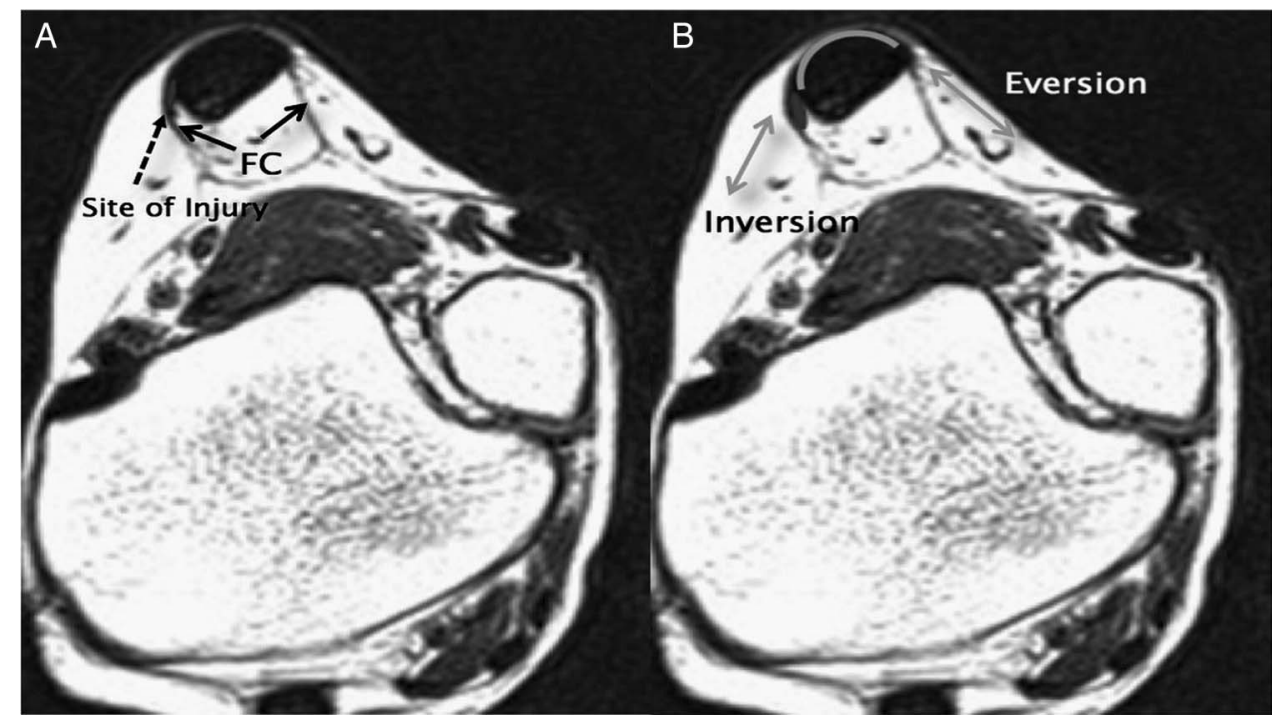

Figure $5 \mathrm{MRI}$ of lower leg showing (A) attachment of the fascia cruris (FC) and the site of injury and (B) possible influence of foot inversion/ eversion. 
Table 2 Time to presentation and initial scan and time to return to play

\begin{tabular}{ll}
\hline Onset to scan/weeks & Return to play/weeks \\
\hline 3 & 6 \\
3 & $12^{*}$ \\
4 & 3 \\
3 & 3 \\
3 & 3 \\
$2 \dagger$ & 1 \\
8 & 1 \\
12 & $22^{*}$ \\
0.5 & 2 \\
1 & 2 \\
$15 \dagger$ & 2 \\
\hline *Participants requiring injection. & \\
†Participants with coexisting US tendinopathy. &
\end{tabular}

pain accurately. Whether it has implications for the development of other Achilles tendon pathology is as yet unknown but warrants further investigation, as are prospective studies to clarify the incidence and confirm the aetiology and the optimum treatment regime. Acute tears of the fascia cruris need to be considered as a cause of Achillodynia with a sudden onset of pain in the active individual. The condition provides a differential diagnosis to paratenonitis.

\section{What are the new findings?}

Tears of the fascia cruris can occur at the attachment to the Achilles tendon and the paratenon.

- The history is usually of acute onset Achillodynia with pain, swelling and tenderness along the medial or lateral border of the tendon in the mid-portion.

- Diagnosis is made using diagnostic ultrasound. Tilting the probe may be required to visualise the fascia cruris attachment to the paratenon.

\section{How might it impact on clinical practice in the near future?}

- Recognition of a new pathological diagnosis in acute onset Achillodynia.

- Differential diagnosis to paratenonitis but different mechanism and imaging appearances.

Acknowledgements Mr M Carmont and Dr A Stecco for use of images.

Contributors NW planned the retrospective review, performed the clinical work for the cases and led the writing of the paper and submitted the study. DM reviewed the work done for the literature review and contributed to the analysis and writing of the paper. KS provided the literature review for the background to the study. OC reviewed the imaging studies independently, provided some images and reviewed the article.

\section{Competing interests None.}

Ethics approval University of Brighton, School of Sport and Service Management Ethics and Governance Committee.

Provenance and peer review Not commissioned; externally peer reviewed.

Open Access This is an Open Access article distributed in accordance with the Creative Commons Attribution Non Commercial (CC BY-NC 4.0) license, which permits others to distribute, remix, adapt, build upon this work non-commercially, and license their derivative works on different terms, provided the original work is properly cited and the use is non-commercial. See: http://creativecommons.org/ licenses/by-nc/4.0/

\section{REFERENCES}

1 De Jonge $S$, van den Berg C, de Vos RJ, et al. Incidence of midportion Achilles tendinopathy in the general population. Br J Sports Med 2011;45:1026-8.

2 Järvinen TAH, Kannus P, Maffulli N, et al. Achilles tendon disorders: etiology and epidemiology. Foot Ankle Clin 2005;10:255-66.

3 Stecco C, Pavan PG, Porzionato A, et al. Mechanics of crural fascia: from anatomy to constitutive modelling. Surg Radiol Anat 2009;31:523-9.

4 Saxena A, Bareither D. Magnetic resonance and cadaveric findings of the "watershed band" of achilles tendon. J Foot Ankle Surg 2001;40:132-6.

5 Soiia K, Karjalainen P, Aronen HJ, et al. High-resolution MR imaging of the asymptomatic achilles tendon: new observations. AJR Am J Roentgenol 1999:173:323-8.

6 Carmont MR, Highland AM, Rochester JR, et al. An anatomical and radiological study of the fascia cruris and paratenon of the Achilles tendon. Foot Ankle Surg 2011:17:186-92.

7 Jarde 0 , Havet $E$, Mertl $P$, et al. [Surgical treatment of chronic Achilles tendiopathies. Report of 52 cases]. Rev Chir Orthop Reparatrice Appar Mot 2000;86:718-23

8 Lehto MU, Järvinen M, Suominen P. Chronic Achilles peritendinitis and retrocalcanear bursitis. Long-term follow-up of surgically treated cases. Knee Surg Sports Traumatol Arthrosc 1994;2:182-5.

9 Nelen G, Martens M, Burssens A. Surgical treatment of chronic Achilles tendinitis. Am J Sports Med 17:754-9 http://www.ncbi.nlm.nih.gov/pubmed/2624286 (accessed 27 Apr 2014).

10 Franklyn-Miller A, Falvey E, McCrory P. Fasciitis first before tendinopathy: does the anatomy hold the key? Br J Sports Med 2009:43:887-9.

11 Kvist $H$, Kvist $M$. The operative treatment of chronic calcaneal paratenonitis. J Bone Jt Surg 1980;62:353-7.

12 ESoS. R. Musculoskeletal Ultrasound Technical Guidelines VI. Ankle. 2013. http:// www.essr.org/html/img/pool/ankle.pdf. (accessed 22 Apr 2014).

13 Schneider C, Klein P, Stolt P, et al. A homeopathic ointment preparation compared with $1 \%$ diclofenac gel for acute symptomatic treatment of tendinopathy. Explore 2005; 1:446-52

14 Stergioulas A, Stergioula M, Aarskog R, et al. Effects of low-level laser therapy and eccentric exercises in the treatment of recreational athletes with chronic achilles tendinopathy. Am J Sports Med 2008;36:881-7.

15 Mueller-Wohlfahrt H-W, Haensel L, Mithoefer K, et al. Terminology and classification of muscle injuries in sport: the Munich consensus statement. $\mathrm{Br} J$ Sports Med 2013:47:342-50.

16 Webborn N, Webborn N. A Neuropathic model to the etiology and management of Achilles tendinopathy. In: Woo S, Renstrom P, Arnoczky P. eds. Tendinopathy in athletes. Oxford: Blackwell Publishing, 2007:145-59.

17 Webborn ADJ. Novel approaches to tendinopathy. Disabil Rehabil 2008;30: 1572-7.

18 Fredberg U. Local corticosteroid injection in sport: review of literature and guidelines for treatment. Scand J Med Sci Sports 1997;7:131-9.

19 Coombes BK, Bisset L, Vicenzino B. Efficacy and safety of corticosteroid injections and other injections for management of tendinopathy: a systematic review of randomised controlled trials. Lancet 2010;376:1751-67. 